\title{
Role of pheromones and its antioxidants in IPM
}

\begin{abstract}
Pheromone technology has potential to add value in long-term pest management of many economically important pests. Inclusion of insecticides or sterilant in pheromone formulations may not be major obstacles to public acceptance in urban areas. Pheromone technology is cost effective and applicable in area wide pest management approach.
\end{abstract}

Volume 6 Issue 2 - 2017

\author{
Divya C,' Hiren G Kanara, ${ }^{2}$ Jethva DM ${ }^{3}$ \\ 'Department of Entomology, India \\ ${ }^{2}$ Assistant Research Scientist, Grassland Research Station, India \\ ${ }^{3}$ Dhari and Associate Research Scientist, Biocontrol Research \\ Laboratory, India
}

Correspondence: Divya C, Department of Entomology, India, Email divuentomology@gmail.com

Received: October 12, 2016 | Published: January 30, 2017

\section{Introduction}

Insects rely more heavily on chemical signals than on any other form of communication. Semio chemicals or info chemicals serves as a form of language that helps to mediate interactions between organisms. Semio chemicals are of two types namely pheromones and allele chemicals. Pheromones are exocrine secretions of insects which are used for communication among different individuals of the species. Pheromones are "Semiochemicals used for intra specific communication between individuals of a single species". ${ }^{1}$ Insect pheromones fall into several categories viz., change in insects (Primer and Releaser), functional group (Type- I \& II) and number of components (Mono \& Multi component).

\section{Different types of pheromone traps}

According to the studies conducted by Karuppuchamy et al.,2 in Tamil Nadu with gossyplure revealed that water pan traps were superior to sticky delta traps in catching more male moths. Among the water pan traps used, yellow pan traps caught more males followed by black tray and red pan traps. The peak male activity was found between cotton crop was 120days old (December) to 180days old (February-March). The effectiveness of sticky delta traps was increased by changing the side of the traps.

Taneja et al., ${ }^{3}$ reported that mmaximum number of moth catches was obtained when rubber stopper was used as a dispenser and cotton seed oil as a moth sticking material.

\section{Factors affecting the pheromone trap}

Taneja et al., ${ }^{3}$ reported that the effectiveness of pheromone lure against $P$. gossypiella is affected by number of factors viz., ratio of component pheromones; trap design; height and distance and wind velocity. The trap installed at plant canopy level with 60meters distance between two traps was effective to capture more number of moths compared to 40 and 20 meter distance. Athanassiou et al. ${ }^{4}$ evaluated the various trap designs and quantity of lure for trapping of pink boll worms and observed that the funnel trap was very effective to trap higher number of moths whereas the trap baited with one lure was sufficient to capture higher number of moths.

\section{Antioxidants}

Yuxiu Liu et al., ${ }^{5}$ suggested that persistence and catching efficacy of synthetic chemicals was improved by addition of the antioxidant BHT. The optimum percentage was obtained at 5-10\% BHT. The data also exhibited that high percentage of BHT (20\%) inhibited the attractiveness of pheromone chemicals.

\section{Pheromone as component of IPM}

\section{Monitoring and mass trapping}

Ghodki et al., ${ }^{6}$ reported that the high moth catches (432 catches/ trap/week); maximum eggs and larval population per plant during 48th meteorological week (November-December). A field studies on population dynamics of $P$. gossypiella based on moth catches in pheromone trap at Junagadh revealed that its population gradually increased from first week of October, reached the pick in third week of December and decreased gradually in the later period of cotton crop. ${ }^{7}$

\section{Mating distruption}

Boguslawaski et al., ${ }^{8}$ reported that organic cotton with mating disruption had less infestation of pink bollworm larvae as compared to conventional cotton, with or without insecticide.

\section{Effectiveness of pheromone traps over other protection measures}

Dhawan et al., ${ }^{9}$ observed that gossyplure @ 10g ai/ha can increase the effectiveness of quinalphos $50 \mathrm{~g}$ ai $/$ ha or cypermethrin $50 \mathrm{~g}$ ai $/ \mathrm{ha}$ for pink bollworms control and gave higher yield than that of the insecticides used alone.

Gupta et al., ${ }^{10}$ found that application of sex pheromone lures for all the three bollworms (Pink bollworms, American bollworms and Spotted bollworms) in trap @ 10/ha and six sprays of insecticides resulted in less shedding of fruiting bodies, damage to locule and bolls and higher net profit.

\section{Conclusion}

Pheromone technology has potential to add value in long-term pest management of many economically important pests. Inclusion of 
insecticides or sterilant in pheromone formulations may not be major obstacles to public acceptance in urban areas. Pheromone technology is cost effective and applicable in area wide pest management approach.

\section{Future thrust}

The cocktail approach i.e., putting together several pheromones in one device or treatments has not been successful and more studies should be conducted in order to get a better understanding of the concept. The current approach therefore is that one has to focus attention on synthesizing large quantities of pheromones for the farmers' use by producing them indigenously at a highly competitive economic price. Further, effectively integrating pheromone application into an IPM strategy where mixture of specific and general pest control application are used will be a sustainable approach and deserves to be tested.

\section{Acknowledgements}

None.

\section{Conflict of interest}

The author declares no conflict of interest.

\section{References}

1. Karlson P, Butenandt A. Ann Rev Ent. 1959;4:39-59.

2. Karuppuchamy P, Balasubramanian M. Field evaluation of gossyplure the synthetic sex pheromone of pectinophora gossypiella in Tamil nadu. Indian J Ent. 1990;52(2):107-179.
3. Taneja SL, Jayaswal AP. Factors affecting male pink bollworm moth catches in gossyplure baited traps. J Plant Prot. 1983;11:78-83.

4. Athanassiou CG, Kavallieratos NG, Gravanis FT, et al. Influence of trap type, pheromone quantity and trapping location on capture of the pink bollworm, Pectinophora gossypiella (Saunders) (Lepidoptera: Gelechiidae). Appl Entomol Zool. 2002;37(3):385-391.

5. Yuxiu Liu, Xianzuo Meng. Trapping effect of synthetic sex pheromone of acleris fimbriana (lepidoptera: tortricidae) in Chinese northern orchard. $Z$ Naturforsch. 2002;58c:421-425.

6. Ghodki BS, Hage ND. Correlation studies on pheromone trap catches of pink bollworm with its field infestation. Insect Environment. 2006;12(2):78-80.

7. Anonymous. Annual Report-Plant Protection sub-committee. Junagadh: GAU; 2005. p. 12-13.

8. Boguslawaski CV, Basedow T. Appl Ent Studies in cotton fields in Egypt on the effects of pheromone mating distribution on pink bollworm on the occurrence of other arthropods. 2001;125:327-331.

9. Dhawan AK, Simwat GS. Field evolution of some botanical insecticides alone or in combination wuth other insecticides for management of bollworm complex in cotton. Ind J of Agri Sci. 1993;63(3):193-194.

10. Gupta MP, Shrivastava SK. Integrated pest management. Ind J Agri Sci. 1998;67(5):261-263. 